OPEN ACCESS

Edited by:

Xuelei Ma,

Sichuan University, China

Reviewed by:

Michael Orth,

University Hospital LMU Munich,

Germany

Michael Hader,

University Hospital Erlangen, Germany

*Correspondence:

Linlin Wang

wanglinlinatin@163.com

Specialty section:

This article was submitted to

Cancer Immunity and

Immunotherapy,

a section of the journal

Frontiers in Immunology

Received: 08 November 2020 Accepted: 02 March 2021

Published: 30 March 2021

Citation:

Li B, Jiang C, Pang L, Zou B, Ding $M$,

Sun $X, Y u J$ and Wang L (2021)

Toxicity Profile of Combining

PD-1/PD-L1 Inhibitors and Thoracic

Radiotherapy in Non-Small Cell Lung

Cancer: A Systematic Review.

Front. Immunol. 12:627197.

doi: 10.3389/fimmu.2021.627197

\section{Toxicity Profile of Combining PD-1/PD-L1 Inhibitors and Thoracic Radiotherapy in Non-Small Cell Lung Cancer: A Systematic Review}

\author{
Butuo Li ${ }^{1}$, Chao Jiang ${ }^{2}$, Linlin Pang ${ }^{1}$, Bing Zou ${ }^{1}$, Mingjun Ding ${ }^{1,3}$, Xindong Sun ${ }^{1}$, \\ Jinming $Y u^{1}$ and Linlin Wang ${ }^{1 *}$ \\ ${ }_{1}^{1}$ Department of Radiation Oncology, Shandong Cancer Hospital and Institute, Shandong First Medical University and \\ Shandong Academy of Medical Science, Jinan, China, ${ }^{2}$ Department of Otorhinolaryngology \& Head and Neck Surgery, \\ Shandong Provincial Hospital Affiliated to Shandong First Medical University, Jinan, China, ${ }^{3}$ Department of Radiation \\ Oncology, Shandong First Medical University and Shandong Academy of Medical Sciences, Tai'an, China
}

Background: The combination of immune checkpoint inhibitors (ICls) and thoracic radiotherapy (TRT) has shown significant clinical activity in patients with non-small cell lung cancer (NSCLC). However, the currently available data on adverse events (AEs) were derived from a small subset of patients included in prospective clinical trials or retrospective studies. Thus, we conducted this systematic review to determine the AEs associated with this combination treatment.

Methods: An electronic literature search was performed in databases and conference proceedings of prospective clinical trials assessing the combination of ICls and TRT for patients with NSCLC. The systematic analysis was conducted to determine the profile and incidence of AEs of combination treatment. We further performed the comparison of AEs between programmed cell death 1 (PD-1) and programmed cell death ligand 1 (PD-L1) inhibitors, and sequential and concurrent administration of ICls and TRT to help identify high risk patients. The systematic analyses were conducted with the Review Manager (version 5.3; The Cochrane Collaboration, Oxford, United Kingdom) and Stata version 12.0 (StataCorp, College Station, TX, USA) software.

Results: Eleven clinical trials involving 1,113 patients with NSCLC were eligible for analysis. The incidence of all-grade AEs was 95.5\%; that of high-grade AEs (grade $\geq 3$ ) was $30.2 \%$. The most frequent all-grade AE was fatigue (49.7\%), while pneumonitis was the most common high-grade $\mathrm{AE}(3.8 \%)$ and grade $5 \mathrm{AE}(0.6 \%)$. Notably, the toxicity profiles of PD-1 and PD-L1 inhibitors were similar. Concurrent treatment was associated with a higher incidence of higher-grade AEs (41.6\% vs 24.8\%, $\mathrm{P}=0.17)$ and pneumonitis (7.1\% vs 3.9\%, $P=0.14$ ) compared to sequential treatment, but no significant difference was observed.

Conclusion: Most AEs of this combination treatment are tolerable; as the most common high-grade $\mathrm{AE}$, pneumonitis deserves the utmost attention of physicians. The toxicity 


\section{profiles of patients receiving PD-1 or PD-L1 were similar, and no significant difference was observed between concurrent and sequential treatment.}

Keywords: toxicity profile, safety, immunotherapy, immune checkpoint inhibitors, thoracic radiotherapy, systemic analysis

\section{INTRODUCTION}

Cancer immunotherapy targets immunosuppressive molecules, such as programmed cell death 1 /programmed cell death ligand 1 (PD-1/PD-L1) and cytotoxic T-lymphocyte associated antigen 4 (CTLA-4). These immune checkpoint inhibitors (ICIs) were successfully used for the treatment of patients with non-small cell lung cancer (NSCLC) of all stages and shown significant clinical activity and marked efficacy (1-3). This type of therapy has been approved by the US Food and Drug Administration for both first-and second-line treatment of metastatic NSCLC, based on significant improvements in overall response rate, progressionfree survival (PFS), and overall survival (OS) (1-6). In addition, radiotherapy (RT) is also an important treatment modality for lung cancer, exerting its effects by damaging the DNA of tumor cells (7). Importantly, RT has also been recognized as an immune modulator (8). It can not only function as an "in-situ vaccine" by increasing the presentation of tumor-specific antigens (9), but also modulates the local tumor environment, resulting in an enhanced immune response (10).

Multiple preclinical studies have suggested a synergistic activity between ICIs and RT, by inducing the activation and recruitment of more antitumor effector T cells $(11,12)$, as well as the modulation of the tumor immune microenvironment (from "cold" tumor to "hot" tumor) (13-15). In addition, it has been indicated that the synergistic activity of ICIs and RT translates into prolonged survival and abscopal effect in preclinical animal models $(16,17)$. Furthermore, recent clinical trials also suggested the amplified antitumor effect of combination of ICIs and thoracic radiotherapy (TRT) in patients with NSCLC. The secondary analysis of 98 metastatic NSCLC patients treated with pembrolizumab in Keynote-001 trial compared patients who received previous RT with those who did not. The results revealed significantly prolonged PFS (4.4 vs. 2.1 months, respectively, $\mathrm{P}=0.019)$ and $\mathrm{OS}(10.7$ vs. 5.3 months, respectively, $\mathrm{P}=0.026$ ) in the former group (18). The PACIFIC trial performed the comparison of durvalumab against placebo after definitive chemoradiation for stage III NSCLC. Treatment with durvalumab was associated with significant improvements of PFS (17.2 vs 5.6 months, respectively, $\mathrm{P}<0.001)$ and $\mathrm{OS}(28.3$ vs. 16.2 months, respectively, $\mathrm{P}<0.001$ ) (6).

Of note, the synergistic effect of combining TRT and ICIs through modulation of the immune response may also affect the spectrum, incidence, and severity of treatment-related AEs. By targeting T cell negative feedback loops, the ICIs can impair the immune tolerance of the tumor and induce the infiltration of immune cells in normal tissues, resulting in autoimmune disease or syndromes and distinctive toxicity profiles, such as pneumonitis and thyroid dysfunction $(19,20)$. RT may cause a wide range of AEs through the ionizing radiation-induced DNA damage and subsequent inflammation on normal tissues, including pneumonitis, mucositis, esophagitis, fibrosis (particularly in lung tissue), and others (21).

Owing to a certain degree of overlap of the toxicity mechanism and spectrum, the combination of ICIs and TRT may exacerbate the toxicity in patients with NSCLC, particularly pneumonitis. Both the Keynote-001 and PACIFIC studies indicated a higher incidence of all-grade pneumonitis in patients who received the combination therapy. Nevertheless, the risk of developing high-grade pneumonitis did not increase significantly $(6,18)$. Importantly, the available evidence regarding the AEs of the combination of ICIs with TRT is limited and derived from a small subset of patients included in prospective clinical trials or retrospective studies (22).

An enhanced understanding of the spectrum and severity of toxicity would enable better prevention and management of the AEs of this combination therapy, thereby informing the clinical application and design of prospective trials. This systematic review focused on prospective clinical trials assessing the AEs of combination of ICIs with TRT in patients with NSCLC, in order to provide a complete toxicity profile and investigate the incidence of AEs of combination treatment. Notably, the treatment sequence, type of ICIs and RT was thought to have impact on the occurrence of toxicity of combination therapy. We further evaluated the role of different ICIs or treatment sequence on the incidence of AEs, so as to help identify high risk patients and guide the clinical administration of combination of ICIs and TRT.

\section{MATERIALS AND METHODS}

\section{Study Search and Inclusion Criteria}

A comprehensive and methodical literature search was conducted to identify all prospective clinical trials investigating the combination of ICIs and TRT for patients with NSCLC. Data searches were conducted in databases, including PubMed, Embase, and the Cochrane database, from January 2000 to November 2020. Keywords included NSCLC, RT, immune checkpoint, PD-1, PD-LI, and specific ICIs drug names. Clinical trials that met the following inclusion criteria were taken into account: (1) patients with histologically confirmed NSCLC; (2) NSCLC patients receiving combination of ICIs and TRT treatment; (3) studies reporting AEs; (4) studies published in English. Retrospective studies were excluded in order to minimize the risk of bias. Abstracts and presentations were also reviewed to identify relevant clinical trials from major conference proceedings, including the American Society of Clinical Oncology, European Society of Medical Oncology, and 
American Society for Radiation Oncology Annual Meeting, between 2010 and 2020. The detailed information of the search strategy for the eligible studies is presented in the flow diagram according to PRISMA. All studies identified by the search strategy that met the eligibility criteria were evaluated by two independent reviewers.

\section{Data Extraction and Statistical Analysis}

The following information was extracted from each study: National Clinical Trial number, first author, year of publication, phase of the trial, number of patients available for the analysis, age, gender, smokers, histology, line of therapy, type and dose of ICIs drugs, control groups, patterns of combination of ICIs and TRT, dose and segmentation of radiation, and number and incidence of AEs of interest (including fatigue, respiratory system, gastrointestinal tract, skin, and endocrine system toxicities). Newcastle-OttawaScale (NOS) evaluation was performed to assess the quality of included studies. All data were independently reviewed and extracted by two investigators.

Some degree of heterogeneity was expected; thus, the data on AEs extracted from the studies were analyzed using DerSimonian and Laird random effect models. The inverse variance method was used to calculate the pooled incidence of AEs and their 95\% confidence interval (CI). Statistical heterogeneity was evaluated with the Cochrane chi-squared test and $\mathrm{I}^{2}$ statistics. The publication bias was assessed by Egger's linear regression test and funnel plots recommended by the Cochrane Collaboration. $\mathrm{P}<0.05$ was defined as significant publication bias, then non-parametric "trim-and-fill" method was performed to minimize the influence of publication bias on the results. The $\mathrm{Z}$ test was used to compare the AEs linked to PD-1 and PD-L1 inhibitors, as well as the sequential and concurrent administration of ICIs and TRT. All analyses were performed using the Review Manager (version 5.3; The Cochrane
Collaboration, Oxford, United Kingdom) and Stata version 12.0 (StataCorp, College Station, TX, USA) software. Differences were considered statistically significant at $\mathrm{P}<0.05$.

\section{RESULTS}

\section{Characteristics of Eligible Studies}

A total of 623 studies were retrieved and reviewed from the database searches. Of those, 56 duplicate studies were excluded. After careful screening and assessment, 11 clinical trials involving 1,113 patients with NSCLC were finally included in the analysis $(6,23-33)$. Figure 1 illustrates the flow diagram of study selection.

The main characteristics of the included studies are summarized in Table 1. The NOS of included studies ranged from 6-8. There was 10 phase II trials and one phase III trial. Notably, PD-1 inhibitors were utilized in eight trials and PD-L1 inhibitors were utilized in three trials. Sequential administration of ICIs and RT was performed in eight trials, while concurrent therapy was performed in five trials. Patients from 9 trials received only conventional fractionated $\mathrm{RT}$, while patients in one trial received only stereotactic body RT.

\section{Incidence of All-Grade AEs of Interest}

The incidence of all-grade AEs in patients treated with ICIs and TRT was 95.5\% (95\% CI: 91.2-99.8\%). The Egger's test indicated that no significant publication bias existed except for all-grade fatigue $(\mathrm{P}=0.03)$. Then "trim-and-fill" analysis was conducted to addressed the bias, and fatigue was found to be the most frequent AE with the incidence of $49.7 \%$ (95\% CI: 32-67.4\%). AEs of the respiratory system were the second common, and the incidence of cough, dyspnea, and pneumonitis was $43.3 \%, 34.1 \%$, and $23 \%$,

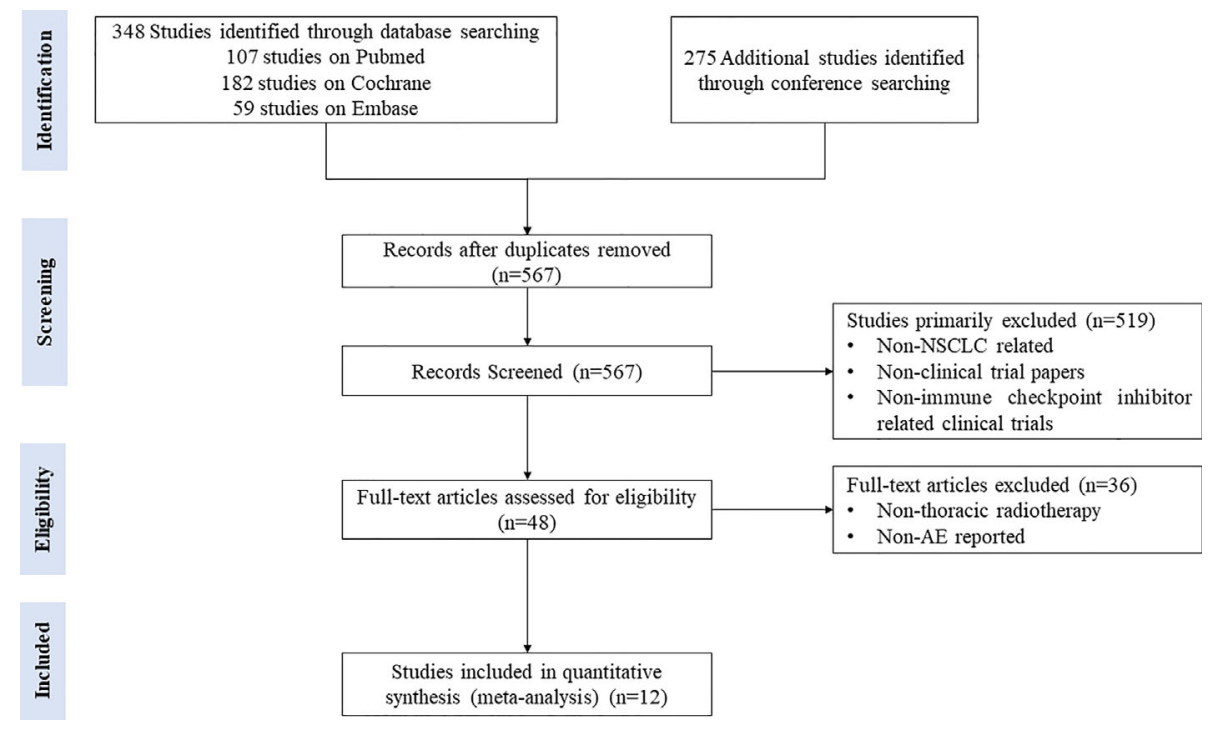

FIGURE 1 | Flow diagram of study inclusion. 


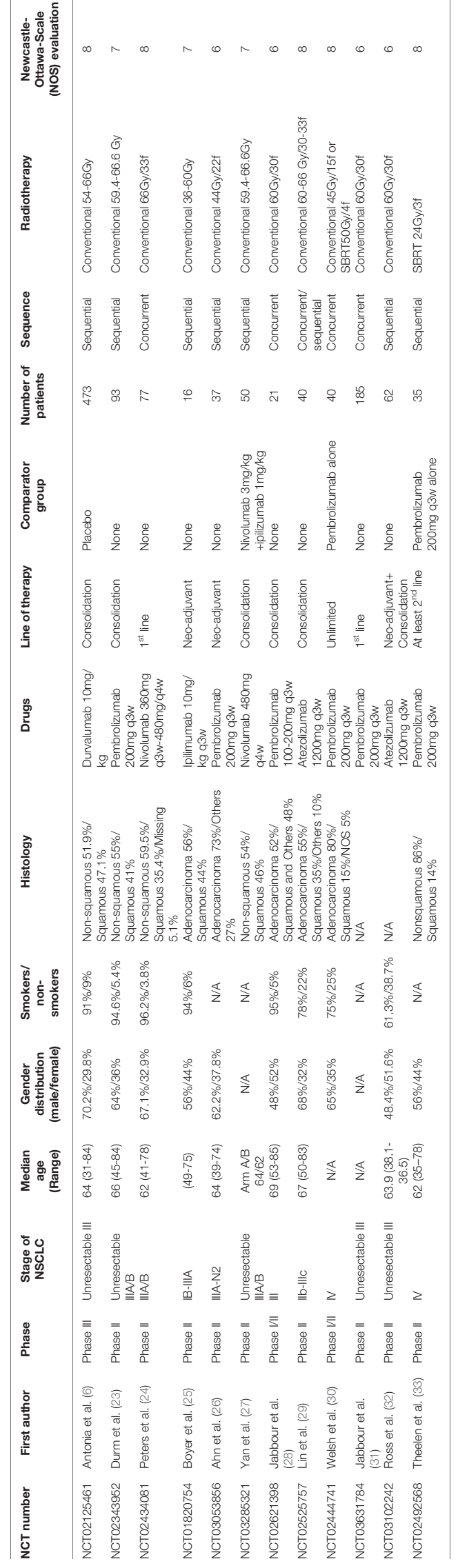

respectively. The funnel plots of all-grade fatigue and pneumonitis were shown in Supplementary Figure 1. Among those who received ICIs and TRT, nausea and diarrhea (AEs related to the gastrointestinal tract) occurred in $29.1 \%$ and $15.8 \%$ of patients, respectively. Of note, the incidence of pruritus, dermatitis, rash, and thyroiditis was $12.4 \%, 11.2 \%, 13.4 \%$, and $9.4 \%$, respectively (Table 2 ).

\section{Incidence of High-Grade AEs of Interest}

Table 3 represents the incidence of high-grade (grade $\geq 3$ ) AEs in patients treated with ICIs and TRT. The funnel plots of highgrade AEs and pneumonitis were shown in Supplementary Figure 1. The Egger's test indicated the publication bias of high-grade fatigue, dyspnea, pneumonitis, nausea, colitis, and rash, and "trim-and-fill" analysis was conducted to address the bias and calculate the pooled incidence. The incidence of highgrade AEs among all patients was 30.2\% (95\% CI: $18.2-42.1 \%$ ). Pneumonitis was the most common high-grade AE $(3.8 \%$, 95\% CI: $2.0-6.9 \%)$, followed by dyspnea (2.1\%) and colitis ( $0.5 \%)$. Besides, the incidence of high-grade fatigue, cough, nausea and colitis was $0.3 \%, 0.3 \%, 0.1 \%$ and $0.5 \%$, respectively. Notably, the incidence of grade 5 AEs was $1.5 \%$ (95\% CI: $0-3.1 \%$ ), and pneumonitis also exhibited the highest incidence $(0.6 \%, 95 \%$ CI: $0.1-1.1 \%)$.

TABLE 2 | Incidence of all-grade AEs of interest.

\begin{tabular}{lccccc}
\hline & Incidence & $\mathbf{9 5 \%} \mathbf{C l}$ & Heterogeneity & $\boldsymbol{\chi}^{\mathbf{2}}$ & $\begin{array}{c}\text { Egger } \\
\text { test } \mathbf{P}\end{array}$ \\
\hline All-grade & $95.5 \%$ & $91.2 \%-99.8 \%$ & $64.1 \%$ & 5.56 & 0.48 \\
AEs & & & & & \\
Fatigue & $49.7 \%$ & $32 \%-67.4 \%$ & $95.2 \%$ & 126.3 & 0.03 \\
Cough & $43.3 \%$ & $25.2 \%-61.5 \%$ & $96.5 \%$ & 170.1 & 0.46 \\
Dyspnea & $34.1 \%$ & $21.8 \%-46.4 \%$ & $91.6 \%$ & 71.49 & 0.17 \\
Pneumonitis & $23 \%$ & $14.2 \%-31.7 \%$ & $85.6 \%$ & 48.4 & 0.27 \\
Nausea & $29.1 \%$ & $15.8 \%-42.5 \%$ & $94.8 \%$ & 115.8 & 0.13 \\
Diarrhea & $15.8 \%$ & $9.8 \%-21.7 \%$ & $72.1 \%$ & 17.9 & 0.89 \\
Rash & $13.4 \%$ & $9.4 \%-17.5 \%$ & $37.6 \%$ & 8.02 & 0.40 \\
Dermatitis & $11.2 \%$ & $0 \%-22.6 \%$ & $85.3 \%$ & 13.6 & 0.11 \\
Pruritus & $12.4 \%$ & $9.4 \%-15.3 \%$ & $14.7 \%$ & 4.69 & 0.63 \\
Thyroiditis & $9.4 \%$ & $3.3 \%-15.4 \%$ & $90.3 \%$ & 61.7 & 0.1 \\
\hline
\end{tabular}

TABLE 3 | Incidence of high-grade AEs of interest.

\begin{tabular}{|c|c|c|c|c|c|}
\hline & Incidence & $95 \% \mathrm{Cl}$ & Heterogeneity & $\chi^{2}$ & $\begin{array}{c}\text { Egger test } \\
P\end{array}$ \\
\hline $\begin{array}{l}\text { High-grade } \\
\text { AEs }\end{array}$ & $30.2 \%$ & $\begin{array}{l}18.2 \%- \\
42.1 \%\end{array}$ & $93.5 \%$ & 122.2 & 0.97 \\
\hline Fatigue & $0.3 \%$ & $0-1.8 \%$ & $52.9 \%$ & 25.9 & 0.02 \\
\hline Cough & $0.3 \%$ & $0-0.8 \%$ & 0.0 & 0.85 & 0.67 \\
\hline Dyspnea & $2.1 \%$ & $0 \%-4.2 \%$ & $36.3 \%$ & 16.4 & 0.008 \\
\hline Pneumonitis & $3.8 \%$ & $1.1 \%-6.6 \%$ & $80.1 \%$ & 52.2 & 0.009 \\
\hline Nausea & $0.1 \%$ & $0 \%-0.3 \%$ & 0.0 & 0.43 & 0.005 \\
\hline Colitis & $0.5 \%$ & $0-1.3 \%$ & 0.0 & 2.34 & 0.016 \\
\hline Rash & $0.3 \%$ & $0-0.8 \%$ & 0.0 & 6.19 & 0.038 \\
\hline Grade 5 AEs & $1.5 \%$ & $0 \%-3.1 \%$ & $70.8 \%$ & 20.5 & 0.20 \\
\hline Pneumonitis & $\%$ & $0.1 \%-1.1 \%$ & & 5.21 & 0.16 \\
\hline
\end{tabular}




\section{Difference in the Incidence of AEs Between PD-1 and PD-L1 Inhibitors Combined With TRT}

The comparison of AEs between PD-1 and PD-L1 inhibitors combined with TRT is shown in Table 4. In terms of all-grade AEs, fatigue was the most common in the PD-1 inhibitor group and showed a similar incidence to that observed in the PD-L1 inhibitor group (50.2\% vs. $49 \%$, respectively, $\mathrm{P}=0.97$ ). All-grade cough was most frequent in patients with PD-L1 inhibitors and TRT; however, there was no significant difference observed compared with PD-1 inhibitors (60\% vs. $36.6 \%$, respectively, $\mathrm{P}=0.39$ ). Notably, the incidence of pneumonitis was comparable between PD-1 and PD-L1 inhibitors combined with TRT $(20.7 \%$ vs. $30 \%$, respectively, $\mathrm{P}=0.21$ ). Furthermore, we did not find significant differences in the incidence of other AEs.

Moreover, the incidence of high-grade AEs was similar between the PD-1 and PD-L1 inhibitor groups (25.6\% vs. $36.6 \%$, respectively, $\mathrm{P}=0.25$ ). Pneumonitis was the most common high-grade $\mathrm{AE}$ in the $\mathrm{PD}-1$ inhibitor group, and there was no significant difference observed compared with the PD-L1 inhibitor group ( $6 \%$ vs. $3.3 \%$, respectively, $\mathrm{P}=0.18$ ). Besides, there was also no significant difference in the incidence of high-grade fatigue, cough, dyspnea, and rash. In summary, no significant difference of the incidence of AEs was observed in the PD-1 or PD-L1 inhibitors when combined with TRT.

\section{Difference in the Incidence of AEs Between Concurrent and Sequential Administration of ICls and TRT}

Table 5 describes the comparison of the toxicity profile between the concurrent and sequential administration of ICIs and TRT. In terms of all-grade AEs, fatigue was the most common in both groups, and there was no significant difference observed between sequential and concurrent treatment (45.5\% vs. $57.3 \%$, respectively, $\mathrm{P}=0.49)$. Compared with patients receiving

TABLE 4 | Difference in incidence of AEs with PD-1 vs PD-L1 inhibitors combined with thoracic radiotherapy.

\begin{tabular}{|c|c|c|c|}
\hline & PD-1 & PD-L1 & $\mathbf{P}$ \\
\hline \multicolumn{4}{|l|}{ All-grade AEs } \\
\hline Fatigue & $50.2 \%(32.2 \%-68.2 \%)$ & 49\% (0\%-99.1\%) & 0.97 \\
\hline Cough & $36.6 \%(14.5 \%-58.8 \%)$ & $60 \%(11.5 \%-99 \%)$ & 0.39 \\
\hline Dyspnea & $30.6 \%(15.3 \%-46 \%)$ & $44.4 \%(0.2 \%-88.6 \%)$ & 0.56 \\
\hline Pneumonitis & $20.7 \%$ (11\%-30.5\%) & $30 \%(19.1 \%-40.8 \%)$ & 0.21 \\
\hline Nausea & $28.2 \%(8.2 \%-48.2 \%)$ & $33.8 \%(0 \%-74 \%)$ & 0.81 \\
\hline Diarrhea & 13.3\% (5.5\%-21.1\%) & $18.8 \%$ (13.2\%-20.8\%) & 0.20 \\
\hline Thyroiditis & $7.3 \%(1.1 \%-13.6 \%)$ & $11.8 \%(9 \%-14.6 \%)$ & 0.20 \\
\hline Rash & $12.4 \%(7.5 \%-17.3 \%)$ & 18.3\% (3.7\%-32.9\%) & 0.45 \\
\hline Pruritus & $12.6 \%(7.3 \%-17.8 \%)$ & $12.5 \%(9.5 \%-15.5 \%)$ & 0.97 \\
\hline \multicolumn{4}{|c|}{ High-grade AEs } \\
\hline Grade $\geq 3$ AEs & $25.6 \%(5.8 \%-45.4 \%)$ & $36.6 \%$ (19.3\%-53.8\%) & 0.25 \\
\hline Fatigue & $1.5 \%(0 \%-3.2 \%)$ & $5.2 \%(0-17.1 \%)$ & 0.542 \\
\hline Cough & $0.5 \%(0-1.3 \%)$ & $0.4 \%(0-0.9 \%)$ & 0.85 \\
\hline Dyspnea & $3.7 \%(0.7-6.6 \%)$ & $3.1 \%(0 \%-8.3 \%)$ & 0.84 \\
\hline Pneumonitis & $6 \%(2.4 \%-9.6 \%)$ & $3.3 \%(1.7 \%-4.8 \%)$ & 0.18 \\
\hline Rash & $1.6 \%(0-3.4 \%)$ & $0.3 \%(0-0.7 \%)$ & 0.32 \\
\hline
\end{tabular}

TABLE 5 | Difference in incidence of AEs with concurrent vs sequential ICls and thoracic radiotherapy.

\begin{tabular}{lccc}
\hline & Sequential & Concurrent & P \\
\hline All-grade AEs & & & \\
Fatigue & $45.5 \%(26.2 \%-54.8 \%)$ & $57.3 \%(35.1 \%-68.7 \%)$ & 0.49 \\
Cough & $44.5 \%(26.8 \%-62.2 \%)$ & $51.9 \%(13.7 \%-90.1 \%)$ & 0.73 \\
Dyspnea & $24.5 \%(17.5 \%-31.6 \%)$ & $45 \%(16.5 \%-73.4 \%)$ & 0.17 \\
Pneumonitis & $21.3 \%(10.1 \%-32.5 \%)$ & $25.8 \%(9.3 \%-42.2 \%)$ & 0.66 \\
Nausea & $16.4 \%(9.1 \%-23.8 \%)$ & $41.9 \%(10.2 \%-73.6 \%)$ & 0.13 \\
Diarrhea & $18.2 \%(14.2 \%-22.2 \%)$ & $14.1 \%(5 \%-23.3 \%)$ & 0.42 \\
Thyroiditis & $10.2 \%(7.3 \%-13.2 \%)$ & $9 \%(0 \%-21.4 .4 \%)$ & 0.84 \\
Pruritus & $13.5 \%(8.9 \%-18 \%)$ & $10.5 \%(9.5 \%-15.3 \%)$ & 0.42 \\
High-grade AEs & & & \\
Grade $\geq 3$ AEs & $24.8 \%(13.1 \%-36.5 \%)$ & $41.6 \%(22.1 \%-61 \%)$ & 0.17 \\
Fatigue & $1.9 \%(0-4.7 \%)$ & $1.6 \%(0-4.2 \%)$ & 0.89 \\
Cough & $0.5 \%(0-1 \%)$ & $0.4 \%(0-1.4 \%)$ & 0.93 \\
Dyspnea & $4.3 \%(0.2 \%-8.5 \%)$ & $2.1 \%(0-4.2 \%)$ & 0.35 \\
Pneumonitis & $3.9 \%(0.7 \%-7.1 \%)$ & $7.1 \%(4.4 \%-9.7 \%)$ & 0.14 \\
Nausea & $1.3 \%(0-3.1 \%)$ & $0.7 \%(0-2 \%)$ & 0.62 \\
Colitis & $0.8 \%(0-2.0 \%)$ & $0.5 \%(0-1.9 \%)$ & 0.80 \\
\hline
\end{tabular}

sequential ICIs and TRT, those who received concurrent treatment had a slightly higher incidence of all-grade pneumonitis; however, this difference was not statistically significant ( $25.8 \%$ vs. $21.3 \% \%$, respectively, $\mathrm{P}=0.66)$. Although no significant difference was observed, the incidence of other respiratory AEs, including cough and dyspnea, was also higher in concurrent treatment group. Moreover, there were also no significant differences in the incidence of all-grade nausea, thyroiditis, and pruritus between concurrent and sequential ICIs and RT.

Concurrent treatment with ICIs and TRT was related to a slightly higher incidence of high-grade AEs compared with sequential treatment ( $41.6 \%$ vs. $24.8 \%$, respectively, $\mathrm{P}=0.09$ ). Although no significant difference was observed, concurrent ICIs and TRT was associated with higher rate of high-grade pneumonitis compared to sequential treatment $(7.1 \%$ vs $3.9 \%$, $\mathrm{P}=0.14$ ). Besides, the risk of high-grade fatigue, cough, dyspnea, nausea, and colitis was also similar between the concurrent and sequential treatment groups. In summary, the incidence of AEs of patients receiving concurrent ICIs and TRT was comparable to sequential treatment.

\section{DISCUSSION}

The potential synergistic effect of the combination of ICIs and TRT has been reported in several preclinical studies (11-15). According to prospective clinical trials, this effect translates into survival benefit for patients with NSCLC $(1,4-6)$. However, the currently available safety information is primarily based on a limited set of studies. Thus, the present study is the first to systematically characterize the toxicity profiles and demonstrate the safety and tolerability of the combination of ICIs and TRT in patients with NSCLC.

The efficacy of combining TRT and immunotherapy is believed that $1+1$ equal more than 2 (15), whether the synthetic effect of combination treatment would double the 
toxicities remains to be clarified. The potential mechanisms involved in the toxicity associated with this combination treatment are unknown. While both ICIs and TRT have the capacity to evoke toxicities in normal tissues when administered alone, and the synthetic effect may also induce the overlap of the profile and mechanism of toxicity $(34,35)$.

The underlying etiology and mechanisms of AEs associated with ICIs is suggested to be related to the disruption of immunologic homeostasis (36). This results in an immuneboosting effect through a series of processes involving autoreactive lymphocytes, autoantibodies, and cytokines (37, 38). The AEs associated with ICIs are the consequences of excessive immunity against normal tissue, involving autoimmune and pro-inflammatory manifestations in the skin, endocrine, gastrointestinal, respiratory, and cardiovascular systems, etc. (36). In addition, TRT may also cause a wide range of AEs, including pneumonitis, mucositis, esophagitis, fibrosis (particularly in lung tissue), and others $(39,40)$, which is suggested to be induced by the induction of DNA breaks, production of reactive oxygen species (41), and the release of damage-associated molecular patterns (DAMPs) $(42,43)$. These effects lead to subsequent acute inflammation-like pneumonitis, mucositis, and esophagitis in the short term $(44,45)$. And succeeding repair and regeneration processes could manifest as chronic events to drive excessive tissue remodeling, resulting in late-onset toxicity such as fibrosis (45-47). The immunological response and altered microenvironment play a central role in the development of either short- or long- term toxicity related to TRT.

The administration of ICIs could also magnify the inflammatory response in irradiated normal tissue and result in infiltration of redundant immunocytes infiltrating and release of inflammatory factors. Furthermore, there may be a certain degree of overlap between the toxicities of RT and immunotherapy. In theory, the combination of TRT and ICIs should be associated with increased toxicity in patients with NSCLC; yet, the degree of increase remains unclear.

We performed this systematic analysis of 1,113 patients with NSCLC who received treatment with the combination of ICIs and TRT in 11 prospective clinical trials; the incidence of allgrade AEs was $95.5 \%$, while that of high-grade AEs was $30.2 \%$. These rates are higher than those of AEs caused by ICIs monotherapy in a previous meta-analysis $(65.8 \%$ and $16.5 \%$, respectively) (48). As expected, the combination of ICIs and TRT was associated with higher toxicity; however, the observed increase remained within acceptable levels. Even so, stricter screening prior to initiating treatment and closer monitoring during treatment should be performed for NSCLC patients receiving combination of TRT and ICIs, which would help to decrease the incidence of $\mathrm{AE}$ and avoid fatal AE.

Similar to treatment with ICIs, the combination of ICIs and TRT also results in a wide variety of AEs, including fatigue, skin toxicity, and events related to the respiratory system, gastrointestinal tract, and endocrine system. Fatigue was the most frequent among all-grade AEs in patients with NSCLC treated with the combination of ICIs and TRT, which was consistent with the toxicity profile of ICIs $(49,50)$. Besides, colitis, thyroiditis and hepatitis are the common autoimmune disease of ICIs, mediated by cytotoxic $\mathrm{T}$ cells against corresponding organs. Our systemic review found that the incidence of high-grade colitis in NSCLC patients receiving ICIs and TRT was $0.5 \%$, which was similar to the high-grade colitis caused by ICIs monotherapy (0.6\%) (51). And thyroiditis was also well-tolerated with the incidence of $9.4 \%$. Whereas only 2 studies reported the incidence of hepatitis among 11 studies. Ahn et al. reported one cases of grade 3 autoimmune hepatitis among 37 patients $(8.1 \%)$ (26), and Theelen et al. reported that none of 35 patients developed hepatitis (33). The combination of ICIs and TRT didn't significantly expand the incidence of hepatitis compared to ICIs monotherapy (5-10\%) (52). In summary, the outer-pulmonary toxicity of ICIs and TRT was well-tolerated, and was not significantly elevated compared to ICIs monotherapy. Just as ICIs monotherapy, regular measurement of thyroid and liver function is also required during combination treatment. Besides, the occurrence of diarrhea should be alert to colitis, whose symptom may not correlate with colitis severity as seen by endoscopy and histology (53).

The potential mechanisms of outer-pulmonary AEs induced by ICIs may include the similar antigenic epitope and cross-reactivity of T cells against tumor and normal tissue, and elevated cytokines (36). With the joint of RT, the "in-situ vaccination and immunomodulation effect" leads to the increased release of antigen and elevated infiltration of lymphocytes. Then the similar antigenic epitope in normal tissue induced the elevated recruitment of immune cells, and release of cytokines and antibodies, followed by excessive immunity against normal tissue, involving autoimmune and pro-inflammatory manifestations. Due to the limited amount of specific antigen in outer-pulmonary tissues, there was no significant increase of infiltrated immune cells and excessive immunity on normal tissues. Thus, only a slightly increase of related outer-pulmonary AEs was observed in this systemic review, with an incidence of high-grade AEs less than 3\%.

However, the cumulative toxicity of radiation and ICIs could give rise to the higher incidence of inter-pulmonary AEs. The incidence of all-grade cough, dyspnea and pneumonitis was $43.3 \%, 34.1 \%$ and $23 \%$. Thus, the pulmonary function test and routine CT scans prior to initial treatment are recommended to guide the patterns of combination treatment, such as the dose and fraction of TRT. Pneumonitis was the most common among high-grade AEs (3.8\%) and grade 5 AEs (0.6\%), which is higher than that of ICIs monotherapy (52), and associated with increased treatment discontinuation and mortality in NSCLC patients treated with combination therapy $(6,26)$. CT scans should also be performed in the process of treatment to evaluate pneumonitis, and early detection and timely intervention (such as dose adjustment) could decrease the rate of discontinuation of treatment and treatment-related death to a great extent.

The TRT-induced DNA damage contributes to the injury of lung tissue, and is followed by the release of antigen and inflammatory factors (e.g., tumor necrosis factor [TNF] and 
transforming growth factor beta [TGF- $\beta]$ ). And the administration of ICIs unleashes $\mathrm{T}$ cells to kill the tumor and repair normal tissue. Moreover, the recruitment of redundant immunocytes in lung tissue may magnify inflammation and exacerbate the pulmonary toxicity (54). Previous pre-clinical studies also showed that changes in inflammatory and a 2.1fold increase of CD8+ T-cells were observed in irradiated lung tissues of mice receiving RT and ICIs compared with RT alone; however, there was no significant elevation in mortality (55). And elevated TNF, which mediates the synergistic effect of the combination treatment, was also associated with pulmonary toxicity $(13,56)$.

In addition, exposure to smoking and poor condition of the lung due to other diseases (e.g., obstructive pulmonary disease) are related to increased toxicity in patients with NSCLC (57). Also, the presence of tumor burden in the lung may limit the tolerance to injury. Thus, it is recommended that clinicians carefully evaluate the risk of pneumonitis based on the smoking history, pulmonary function test, and others, and allocate more of their attention to prevent, monitor, recognize, and manage pneumonitis at the early stage of treatment with ICIs and TRT. Thorough understanding of the mechanism of toxicity caused by the combination treatment is urgently needed to determine useful biomarkers for the identification of highrisk patients.

The exploration of related factors of toxicity could help to identify high risk patients and enable better prevention and management of the AEs of combination of TRT and ICIs. And the parameters of ICIs drugs or radiotherapy, and sequence of treatment were thought to play important roles on the AEs of combination treatment. At present, there is no head-to-head study to compare the difference in AEs between PD-1 and PDL1 inhibitors combined with TRT. A previous study stated that the toxicity profiles of PD-1 and PD-L1 inhibitors in NSCLC patients are similar (58). And PD-1 inhibitors have been associated with a significantly higher incidence of highgrade immune-related pneumonitis $(1.1 \%$ vs $0.4 \%, \mathrm{P}=0.01)$ (59). The potential mechanism involved in the higher incidence of pneumonitis may be the blockage of PD-1-PDL2 induced by PD-1 inhibitors. This blockage assists in the release of cytokines and proliferation of self-reactive $\mathrm{T}$ cells, leading to the enhancement of the antitumor effect and AEs (60).

When combined with TRT, no significant difference was recorded between $\mathrm{PD}-1$ and $\mathrm{PD}-\mathrm{L} 1$ inhibitor in our systemic review. Notably, the combination of TRT and PD-1 or PD-L1 inhibitors were related to higher incidence of pneumonitis compared to PD-1 or PD-L1 monotherapy. Both ICIs and TRT participated in the development and progression of pneumonitis, and TRT predominated on account of the DNA damage, subsequent inflammatory response, and collagen deposition on normal lung tissue. The leading role of TRT rather than ICIs might be the reason for the similar incidence of PD-1 and PD-L1 inhibitors when combined with TRT. Thus, the selection of candidate ICIs is recommended, primarily depending on their efficacy rather than the toxicity.
Moreover, the role of treatment sequence of TRT and ICIs on the incidence of toxicity was of close concern. Due to the timedependent effect induced by TRT in normal tissue, the toxicity ranges from acute inflammatory effects towards chronic fibrotic side effects $(45,61,62)$. Thus, concurrent or sequential treatment with ICIs and TRT may induce different side effects, particularly in lung tissue. Concurrent treatment is theoretically associated with higher toxicity due to the acute phase inflammation and overlapping toxicity. However, the collective available evidence on the safety of concurrent or sequential treatment with ICIs and TRT is varied. The secondary analysis of the PACIFIC study revealed that patients with NSCLC who received durvalumab within 14 days from the last session of TRT had superior survival and a higher rate of pneumonitis (63). Nevertheless, another retrospective study of 79 patients did not find differences in AEs between the concurrent and sequential administration of ICIs and RT (22).

As expected, this systematic analysis revealed that concurrent administration of ICIs and TRT led to an improved toxicity profile, particularly with regard to pneumonitis; while no statistical significance was found. In addition, the increase of outer-pulmonary AEs by concurrent treatment was not obvious. Thus, pulmonary function test and routine CT scans are essential for NSCLC patients receiving concurrent ICIs and TRT. The potential mechanisms for the statistically undifferentiated incidence of AEs between the concurrent and sequential treatments are unknown, and a hypothesis is provided below. Firstly, previous evidence has demonstrated the "long tail effect" of ICIs on the survival of patients with NSCLC (64-66). While the "long tail effect" and immunological memory of ICIs could also give rise to long-lasting AEs, which may contribute to the increased incidence of AEs when combined with subsequent RT. Moreover, immunotherapy followed by RT was also found to induce radiation recall pneumonitis, which was triggered by a "remembered" and "overreacted" process of the immunomodulatory effect (67). Thus, sequential treatment could not completely avoid the overlapping toxicity and significantly decrease the occurrence of AEs, as initially envisioned. Further studies are warranted to identify the acute and long-term toxicity, as well as the respective mechanisms of different sequences of RT and ICIs combination therapy.

Except for the sequence of treatment, the dose and fraction of TRT were also associated with the toxicity of TRT and ICIs. Welsh et al. performed an exploratory analysis and revealed that the median PFS was better in SBRT group compared to traditional RT (20.8 vs 6.8 months, $\mathrm{P}=0.03$ ) in metastatic NSCLC patients, and 3 and 5 patients experienced high-grade AEs in SBRT and traditional RT group respectively. Based on available data, there was no significant difference on toxicity between two groups (30). However, further studies are needed to assess the difference on efficacy between SBRT and conventional radiotherapy combined with ICIs, especially for metastatic NSCLC patients. In addition, the radiotherapy dose and site for metastatic NSCLC should also be taken into consideration in future studies to evaluate the safety and efficacy of combining ICIs and TRT. 
A limitation of this study is the relatively small number of eligible studies included in our analyses. However, all published clinical trials of the combination of ICIs and TRT in patients with NSCLC were included to capture the safety data. In addition, the assessment of AEs was somewhat subjective and varied between studies. Thus, our analysis depended on the quality of AE reporting by investigators. Moreover, there was heterogeneity among the studies included in this systematic analysis. Further larger scale, multicenter, randomized controlled trials and real-word studies are warranted to evaluate the safety of the combination of ICIs and TRT in patients with NSCLC.

\section{CONCLUSION}

This systematic review, for the first time, draws attention to the toxicity profile of the combination of ICIs and TRT for patients with NSCLC, and focused comprehensive effort at the comparison of AEs based on different ICIs and different treatment settings. Most AEs of the combination treatment are tolerable. Nonetheless, pneumonitis was the most common highgrade $\mathrm{AE}$ and deserves the utmost attention of physicians due to its leading role in AE-related death. Careful selection of patients at high risk and close monitoring for pneumonitis in patients with NSCLC receiving the combination of ICIs and TRT are recommended. This systematic analysis also demonstrated similar safety profiles between PD-1 and PD-L1 inhibitors combined with TRT, and a relatively higher incidence of AEs induced by concurrent treatment. Above all, optimal treatment selection is recommended, primarily depending on the efficacy rather than the safety of the candidate drugs. Furthermore, the identification of patients at high risk of toxicity is necessary prior to the administration of concurrent ICIs and RT. The findings of this comprehensive analysis could lay a foundation to accelerate the development of ICIs and TRT combination treatment, and achieve the goal of maximizing benefit and minimizing toxicity.

\section{REFERENCES}

1. Rittmeyer A, Barlesi F, Waterkamp D, Park K, Ciardiello F, von Pawel J, et al. Atezolizumab versus docetaxel in patients with previously treated non-smallcell lung cancer (OAK): a phase 3, open-label, multicentre randomised controlled trial. Lancet (London England) (2017) 389(10066):255-65. doi: 10.1016/s0140-6736(16)32517-x

2. Horn L, Spigel DR, Vokes EE, Holgado E, Ready N, Steins M, et al. Nivolumab Versus Docetaxel in Previously Treated Patients With Advanced Non-SmallCell Lung Cancer: Two-Year Outcomes From Two Randomized, Open-Label, Phase III Trials (CheckMate 017 and CheckMate 057). J Clin Oncol (2017) 35 (35):3924-33. doi: 10.1200/jco.2017.74.3062

3. Hellmann MD, Paz-Ares L, Bernabe Caro R, Zurawski B, Kim S-W, Carcereny Costa E, et al. Nivolumab plus Ipilimumab in Advanced NonSmall-Cell Lung Cancer. N Engl J Med (2019) 381(21):2020-31. doi: 10.1056/ NEJMoa1910231

4. Borghaei H, Paz-Ares L, Horn L, Spigel DR, Steins M, Ready NE, et al. Nivolumab versus Docetaxel in Advanced Nonsquamous Non-Small-Cell Lung Cancer. N Engl J Med (2015) 373(17):1627-39. doi: 10.1056/ NEJMoa1507643

\section{DATA AVAILABILITY STATEMENT}

The raw data supporting the conclusions of this article will be made available by the authors, without undue reservation.

\section{AUTHOR CONTRIBUTIONS}

Methodology, BL, CJ, LP, and BZ. Software, BL and MD. Validation, XS, JY, and LW. Formal analysis, BL, CJ, BZ, and LW. Data curation, BL, LP, BZ, MD, and XS. Writing-original draft preparation, BL, CJ, LP, and LW. Writing-review and editing, BL, CJ, XS, and LW. Visualization, MD, BZ, JY, and XS. Project administration, XS, JY, and LW. Funding acquisition, JY and LW. All authors contributed to the article and approved the submitted version.

\section{FUNDING}

This work was supported by the following grant: Natural Science Foundation of Shandong Province (Grant No. ZR2019LZL012), the Innovation Project of Shandong Academy of Medical Sciences (2019-04), and the Academic Promotion Program of Shandong First Medical University (2019ZL002).

\section{SUPPLEMENTARY MATERIAL}

The Supplementary Material for this article can be found online at: https://www.frontiersin.org/articles/10.3389/fimmu.2021. 627197/full\#supplementary-material

Supplementary Figure 1 | Funnel plots for (A). All-grade fatigue, (B). All-grade pneumonitis, (C). High-grade AEs, (D). High-grade pneumonitis. Due to the publication bias of all-grade fatigue and high-grade pneumonitis, the figure presents the funnel plot with the missing studies imputed by the trim-and-fill method.

5. Hui R, Garon EB, Goldman JW, Leighl NB, Hellmann MD, Patnaik A, et al. Pembrolizumab as first-line therapy for patients with PD-L1-positive advanced non-small cell lung cancer: a phase 1 trial. Ann Oncol (2017) 28 (4):874-81. doi: 10.1093/annonc/mdx008

6. Antonia SJ, Villegas A, Daniel D, Vicente D, Murakami S, Hui R, et al. Overall Survival with Durvalumab after Chemoradiotherapy in Stage III NSCLC. N Engl J Med (2018) 379(24):2342-50. doi: 10.1056/NEJMoa1809697

7. Santivasi WL, Xia F. Ionizing radiation-induced DNA damage, response, and repair. Antioxid Redox Signal (2014) 21(2):251-9. doi: 10.1089/ars.2013.5668

8. Burnette B, Weichselbaum RR. Radiation as an immune modulator. Semin Radiat Oncol (2013) 23(4):273-80. doi: 10.1016/j.semradonc.2013.05.009

9. Rodriguez-Ruiz ME, Vanpouille-Box C, Melero I, Formenti SC, Demaria S. Immunological Mechanisms Responsible for Radiation-Induced Abscopal Effect. Trends Immunol (2018) 39(8):644-55. doi: 10.1016/j.it.2018.06.001

10. Barker HE, Paget JT, Khan AA, Harrington KJ. The tumour microenvironment after radiotherapy: mechanisms of resistance and recurrence. Nat Rev Cancer (2015) 15(7):409-25. doi: 10.1038/nrc3958

11. Deng L, Liang H, Burnette B, Beckett M, Darga T, Weichselbaum RR, et al. Irradiation and anti-PD-L1 treatment synergistically promote antitumor immunity in mice. J Clin Invest (2014) 124(2):687-95. doi: 10.1172/JCI67313 
12. Sharabi AB, Nirschl CJ, Kochel CM, Nirschl TR, Francica BJ, Velarde E, et al. Stereotactic Radiation Therapy Augments Antigen-Specific PD-1-Mediated Antitumor Immune Responses via Cross-Presentation of Tumor Antigen. Cancer Immunol Res (2015) 3(4):345-55. doi: 10.1158/2326-6066.cir-14-0196

13. Deng L, Liang H, Burnette B, Beckett M, Darga T, Weichselbaum RR, et al. Irradiation and anti-PD-L1 treatment synergistically promote antitumor immunity in mice. J Clin Invest (2014) 124(2):687-95. doi: 10.1172/JCI67313

14. Wirsdorfer F, Cappuccini F, Niazman M, de Leve S, Westendorf AM, Ludemann L, et al. Thorax irradiation triggers a local and systemic accumulation of immunosuppressive CD4+ FoxP3+ regulatory $\mathrm{T}$ cells. Radiat Oncol (London England) (2014) 9:98. doi: 10.1186/1748-717x-9-98

15. Demaria S, Coleman CN, Formenti SC. Radiotherapy: Changing the Game in Immunotherapy. Trends Cancer (2016) 2(6):286-94. doi: 10.1016/ j.trecan.2016.05.002

16. Demaria S, Kawashima N, Yang AM, Devitt ML, Babb JS, Allison JP, et al. Immune-mediated inhibition of metastases after treatment with local radiation and CTLA-4 blockade in a mouse model of breast cancer. Clin Cancer Res (2005) 11(2 Pt 1):728-34.

17. Herter-Sprie GS, Koyama S, Korideck H, Hai J, Deng J, Li YY, et al. Synergy of radiotherapy and PD-1 blockade in Kras-mutant lung cancer. JCI Insight (2016) 1(9):e87415. doi: 10.1172/jci.insight.87415

18. Shaverdian N, Lisberg AE, Bornazyan K, Veruttipong D, Goldman JW, Formenti SC, et al. Previous radiotherapy and the clinical activity and toxicity of pembrolizumab in the treatment of non-small-cell lung cancer: a secondary analysis of the KEYNOTE-001 phase 1 trial. Lancet Oncol (2017) 18 (7):895-903. doi: 10.1016/S1470-2045(17)30380-7

19. Fujii T, Colen RR, Bilen MA, Hess KR, Hajjar J, Suarez-Almazor ME, et al. Incidence of immune-related adverse events and its association with treatment outcomes: the MD Anderson Cancer Center experience. Invest New Drugs (2018) 36(4):638-46. doi: 10.1007/s10637-017-0534-0

20. Weber JS, Yang JC, Atkins MB, Disis ML. Toxicities of Immunotherapy for the Practitioner. J Clin Oncol (2015) 33(18):2092-9. doi: 10.1200/ jco.2014.60.0379

21. Hanania AN, Mainwaring W, Ghebre YT, Hanania NA, Ludwig M. Radiation-Induced Lung Injury: Assessment and Management. Chest (2019) 156(1):150-62. doi: 10.1016/j.chest.2019.03.033

22. von Reibnitz D, Chaft JE, Wu AJ, Samstein R, Hellmann MD, Plodkowski AJ, et al. Safety of combining thoracic radiation therapy with concurrent versus sequential immune checkpoint inhibition. Adv Radiat Oncol (2018) 3(3):391-8. doi: 10.1016/j.adro.2018.05.001

23. Durm GA, Jabbour SK, Althouse SK, Liu Z, Sadiq AA, Zon RT, et al. A phase 2 trial of consolidation pembrolizumab following concurrent chemoradiation for patients with unresectable stage III non-small cell lung cancer: Hoosier cancer research network LUN 14-179. Cancer (2020) 126(19):4353-61. doi: $10.1002 / \mathrm{cncr} .33083$

24. Peters S, Felip E, Dafni U, Belka C, Guckenberger M, Irigoyen A, et al. Safety evaluation of nivolumab added concurrently to radiotherapy in a standard first line chemo-radiotherapy regimen in stage III non-small cell lung cancerThe ETOP NICOLAS trial. Lung Cancer (Amsterdam Netherlands) (2019) 133:83-7. doi: 10.1016/j.lungcan.2019.05.001

25. Boyer MJ, Gu L, Wang X, Kelsey CR, Yoo DS, Onaitis MW, et al. Toxicity of definitive and post-operative radiation following ipilimumab in non-small cell lung cancer. Lung Cancer (Amsterdam Netherlands) (2016) 98:76-8. doi: 10.1016/j.lungcan.2016.05.014

26. Ahn M-J, Park S, Jung HA, Cho JH, Sun J-M, Lee S-H, et al. Phase II, prospective single-arm study of adjuvant pembrolizumab in N2 positive non-small cell lung cancer (NSCLC) treated with neoadjuvant concurrent chemoradiotherapy followed by curative resection: Preliminary results. J Clin Oncol (2019) 37 (15_suppl):8520-. doi: 10.1200/JCO.2019.37.15_suppl.8520

27. Yan M, Durm GA, Mamdani H, Ernani V, Jabbour SK, Naidoo J, et al. Consolidation nivolumab/ipilimumab versus nivolumab following concurrent chemoradiation in patients with unresectable stage III NSCLC: A planned interim safety analysis from the BTCRC LUN 16-081 trial. J Clin Oncol (2020) 38(15_suppl):9010-. doi: 10.1200/ JCO.2020.38.15_suppl.9010

28. Jabbour SK, Berman AT, Decker RH, Lin Y, Feigenberg SJ, Gettinger SN, et al. Phase 1 Trial of Pembrolizumab Administered Concurrently With Chemoradiotherapy for Locally Advanced Non-Small Cell Lung Cancer: A
Nonrandomized Controlled Trial. JAMA Oncol (2020) 6(6):848-55. doi: 10.1001/jamaoncol.2019.6731

29. Lin SH, Lin Y, Yao L, Kalhor N, Carter BW, Altan M, et al. Phase II Trial of Concurrent Atezolizumab With Chemoradiation for Unresectable NSCLC. J Thorac Oncol (2020) 15(2):248-57. doi: 10.1016/j.jtho.2019.10.024

30. Welsh J, Menon H, Chen D, Verma V, Tang C, Altan M, et al. Pembrolizumab with or without radiation therapy for metastatic non-small cell lung cancer: a randomized phase I/II trial. J Immunother Cancer (2020) 8(2):e001001. doi: 10.1136/jitc-2020-001001

31. Jabbour SK, Lee KH, Frost N, Kowalski D, Breder VV, Pollock T, et al. Phase II study of pembrolizumab (pembro) plus platinum doublet chemotherapy and radiotherapy as first-line therapy for unresectable, locally advanced stage III NSCLC: KEYNOTE-799. J Clin Oncol (2020) 38(15_suppl):9008-. doi: 10.1200/JCO.2020.38.15_suppl.9008

32. Ross HJ, Kozono DE, Urbanic JJ, Williams TM, Dufrane C, Bara I, et al. AFT16: Phase II trial of atezolizumab before and after definitive chemoradiation (CRT) for unresectable stage III non-small cell lung cancer (NSCLC). J Clin Oncol (2020) 38(15_suppl):9045-. doi: 10.1200/JCO.2020.38.15_suppl.9045

33. Theelen W, Peulen HMU, Lalezari F, van der Noort V, de Vries JF, Aerts J, et al. Effect of Pembrolizumab After Stereotactic Body Radiotherapy vs Pembrolizumab Alone on Tumor Response in Patients With Advanced Non-Small Cell Lung Cancer: Results of the PEMBRO-RT Phase 2 Randomized Clinical Trial. JAMA Oncol (2019) 5(9):1276-82. doi: 10.1001/ jamaoncol.2019.1478

34. Nishino M, Giobbie-Hurder A, Hatabu H, Ramaiya NH, Hodi FS. Incidence of Programmed Cell Death 1 Inhibitor-Related Pneumonitis in Patients With Advanced Cancer: A Systematic Review and Meta-analysis. JAMA Oncol (2016) 2(12):1607-16. doi: 10.1001/jamaoncol.2016.2453

35. Formenti SC, Demaria S. Systemic effects of local radiotherapy. Lancet Oncol (2009) 10(7):718-26. doi: 10.1016/s1470-2045(09)70082-8

36. Postow MA, Sidlow R, Hellmann MD. Immune-Related Adverse Events Associated with Immune Checkpoint Blockade. N Engl J Med (2018) 378 (2):158-68. doi: 10.1056/NEJMra1703481

37. Osorio JC, Ni A, Chaft JE, Pollina R, Kasler MK, Stephens D, et al. Antibodymediated thyroid dysfunction during T-cell checkpoint blockade in patients with non-small-cell lung cancer. Ann Oncol (2017) 28(3):583-9. doi: 10.1093/ annonc/mdw640

38. Esfahani K, Miller WHJr. Reversal of Autoimmune Toxicity and Loss of Tumor Response by Interleukin-17 Blockade. N Engl J Med (2017) 376 (20):1989-91. doi: 10.1056/NEJMc1703047

39. Ratikan JA, Micewicz ED, Xie MW, Schaue D. Radiation takes its Toll. Cancer Lett (2015) 368(2):238-45. doi: 10.1016/j.canlet.2015.03.031

40. Mavragani IV, Laskaratou DA, Frey B, Candéias SM, Gaipl US, Lumniczky K, et al. Key mechanisms involved in ionizing radiation-induced systemic effects. A current review. Toxicol Res (Camb) (2015) 5(1):12-33. doi: 10.1039/ c5tx00222b

41. Amaravadi RK, Thompson CB. The roles of therapy-induced autophagy and necrosis in cancer treatment. Clin Cancer Res (2007) 13(24):7271-9. doi: 10.1158/1078-0432.CCR-07-1595

42. Ludgate CM. Optimizing cancer treatments to induce an acute immune response: radiation Abscopal effects, PAMPs, and DAMPs. Clin Cancer Res (2012) 18(17):4522-5. doi: 10.1158/1078-0432.CCR-12-1175

43. Hernandez C, Huebener P, Schwabe RF. Damage-associated molecular patterns in cancer: a double-edged sword. Oncogene (2016) 35(46):5931-41. doi: 10.1038/onc.2016.104

44. Schaue D, Micewicz ED, Ratikan JA, Xie MW, Cheng G, McBride WH. Radiation and inflammation. Semin Radiat Oncol (2015) 25(1):4-10. doi: 10.1016/j.semradonc.2014.07.007

45. Simone CB2nd. Thoracic Radiation Normal Tissue Injury. Semin Radiat Oncol (2017) 27(4):370-7. doi: 10.1016/j.semradonc.2017.04.009

46. Yarnold J, Brotons MC. Pathogenetic mechanisms in radiation fibrosis. Radiother Oncol (2010) 97(1):149-61. doi: 10.1016/j.radonc.2010.09.002

47. Tsoutsou PG, Koukourakis MI. Radiation pneumonitis and fibrosis: mechanisms underlying its pathogenesis and implications for future research. Int J Radiat Oncol Biol Phys (2006) 66(5):1281-93. doi: 10.1016/ j.ijrobp.2006.08.058

48. Magee DE, Hird AE, Klaassen Z, Sridhar SS, Nam RK, Wallis CJD, et al. Adverse event profile for immunotherapy agents compared with chemotherapy in solid 
organ tumors: a systematic review and meta-analysis of randomized clinical trials. Ann Oncol (2020) 31(1):50-60. doi: 10.1016/j.annonc.2019.10.008

49. De Velasco G, Je Y, Bosse D, Awad MM, Ott PA, Moreira RB, et al. Comprehensive Meta-analysis of Key Immune-Related Adverse Events from CTLA-4 and PD-1/PD-L1 Inhibitors in Cancer Patients. Cancer Immunol Res (2017) 5(4):312-8. doi: 10.1158/2326-6066.cir-16-0237

50. Khoja L, Day D, Wei-Wu Chen T, Siu LL, Hansen AR. Tumour- and classspecific patterns of immune-related adverse events of immune checkpoint inhibitors: a systematic review. Ann Oncol (2017) 28(10):2377-85. doi: 10.1093/annonc/mdx286

51. Nishijima TF, Shachar SS, Nyrop KA, Muss HB. Safety and Tolerability of PD1/PD-L1 Inhibitors Compared with Chemotherapy in Patients with Advanced Cancer: A Meta-Analysis. Oncologist (2017) 22(4):470-9. doi: 10.1634/ theoncologist.2016-0419

52. Haanen J, Carbonnel F, Robert C, Kerr KM, Peters S, Larkin J, et al. Management of toxicities from immunotherapy: ESMO Clinical Practice Guidelines for diagnosis, treatment and follow-up. Ann Oncol (2017) 28 (suppl_4):iv119-iv42. doi: 10.1093/annonc/mdx225

53. Geukes Foppen MH, Rozeman EA, van Wilpe S, Postma C, Snaebjornsson P, van Thienen JV, et al. Immune checkpoint inhibition-related colitis: symptoms, endoscopic features, histology and response to management. ESMO Open (2018) 3(1):e000278. doi: 10.1136/esmoopen-2017-000278

54. Hwang WL, Pike LRG, Royce TJ, Mahal BA, Loeffler JS. Safety of combining radiotherapy with immune-checkpoint inhibition. Nat Rev Clin Oncol (2018) 15(8):477-94. doi: 10.1038/s41571-018-0046-7

55. Xue J, Du S, Lu Y, Dicker A, Lu B. Abstract 3671: Anti-PD-1 treatment may potentiate the radiation-induced lung injury. Cancer Res (2017) 77(13 Supplement):3671-71. doi: 10.1158/1538-7445.am2017-3671

56. Xue J, Li X, Lu Y, Gan L, Zhou L, Wang Y, et al. Gene-modified Mesenchymal Stem Cells Protect Against Radiation-induced Lung Injury. Mol Ther (2013) 21(2):456-65. doi: 10.1038/mt.2012.183

57. Bouros D, Hatzakis K, Labrakis H, Zeibecoglou K. Association of malignancy with diseases causing interstitial pulmonary changes. Chest (2002) 121 (4):1278-89. doi: 10.1378/chest.121.4.1278

58. Pillai RN, Behera M, Owonikoko TK, Kamphorst AO, Pakkala S, Belani CP, et al. Comparison of the toxicity profile of PD-1 versus PD-L1 inhibitors in non-small cell lung cancer: A systematic analysis of the literature. Cancer (2018) 124(2):271-7. doi: 10.1002/cncr.31043

59. Khunger M, Rakshit S, Pasupuleti V, Hernandez AV, Mazzone P, Stevenson J, et al. Incidence of Pneumonitis With Use of Programmed Death 1 and Programmed Death-Ligand 1 Inhibitors in Non-Small Cell Lung Cancer: A
Systematic Review and Meta-Analysis of Trials. Chest (2017) 152(2):271-81. doi: 10.1016/j.chest.2017.04.177

60. Latchman Y, Wood CR, Chernova T, Chaudhary D, Borde M, Chernova I, et al. PD-L2 is a second ligand for PD-1 and inhibits T cell activation. Nat Immunol (2001) 2(3):261-8. doi: 10.1038/85330

61. Kim JH, Jenrow KA, Brown SL. Mechanisms of radiation-induced normal tissue toxicity and implications for future clinical trials. Radiat Oncol J (2014) 32(3):103-15. doi: 10.3857/roj.2014.32.3.103

62. Rube CE, Uthe D, Schmid KW, Richter KD, Wessel J, Schuck A, et al. Dose-dependent induction of transforming growth factor beta (TGF-beta) in the lung tissue of fibrosis-prone mice after thoracic irradiation. Int $J$ Radiat Oncol Biol Phys (2000) 47(4):1033-42. doi: 10.1016/s0360-3016 (00)00482-x

63. Raben D, Faivre-Finn C, Spigel D, Daniel D, Villegas A, Vincente D, et al. PACIFIC: Overall Survival with Durvalumab versus Placebo after Chemoradiotherapy in Stage III NSCLC. Int J Radiat Oncol Biol Phys (2018) 102(5):1607-8. doi: 10.1016/j.ijrobp.2018.08.057

64. Borcoman E, Kanjanapan Y, Champiat S, Kato S, Servois V, Kurzrock R, et al. Novel patterns of response under immunotherapy. Ann Oncol (2019) 30 (3):385-96. doi: 10.1093/annonc/mdz003

65. Rekers NH, Olivo Pimentel V, Yaromina A, Lieuwes NG, Biemans R, Zegers CML, et al. The immunocytokine L19-IL2: An interplay between radiotherapy and long-lasting systemic anti-tumour immune responses. Oncoimmunology (2018) 7(4):e1414119. doi: 10.1080/2162402x.2017.1414119

66. Chiang AC, Herbst RS. Frontline immunotherapy for NSCLC - the tale of the tail. Nat Rev Clin Oncol (2020) 17(2):73-4. doi: 10.1038/s41571-019-0317-y

67. Chen Y, Huang Z, Xing L, Meng X, Yu J. Radiation Recall Pneumonitis Induced by Anti-PD-1 Blockade: A Case Report and Review of the Literature. Front Oncol (2020) 10:561-61. doi: 10.3389/fonc.2020.00561

Conflict of Interest: The authors declare that the research was conducted in the absence of any commercial or financial relationships that could be construed as a potential conflict of interest.

Copyright (c) 2021 Li, Jiang, Pang, Zou, Ding, Sun, Yu and Wang. This is an openaccess article distributed under the terms of the Creative Commons Attribution License (CC BY). The use, distribution or reproduction in other forums is permitted, provided the original author(s) and the copyright owner(s) are credited and that the original publication in this journal is cited, in accordance with accepted academic practice. No use, distribution or reproduction is permitted which does not comply with these terms. 